\title{
Electron cyclotron wave power loss in fusion plasmas: a model comparison $^{4}$
}

\author{
F Albajar ${ }^{1,5}$, M Bornatici $^{2}$ and F Engelmann ${ }^{3}$ \\ ${ }^{1}$ Asociación EURATOM-CIEMAT, 28040 Madrid, Spain \\ ${ }^{2}$ Physics Department “A. Volta”, University of Pavia, I-27100 Pavia, Italy \\ ${ }^{3}$ Max-Planck-Institut für Plasmaphysik, 85748 Garching, Germany \\ E-mail: ferran.albajar@tech.efda.org
}

\begin{abstract}
For the modelling of hot reactor-grade plasmas a way to compute local electron cyclotron wave losses efficiently and with good accuracy is required. Thereby the approach to calculating the electron cyclotron absorption coefficient is an essential element. A comparison of three available analytical forms (a "quasi-exact" one and the asymptotic forms due to Trubnikov and to Robinson) shows that, overall, the use of Robinson's formula is the most suitable choice.
\end{abstract}

PACS numbers: 52.40.Db, 52.50.-b, 52.55.Dy

Submitted to Nuclear Fusion

\section{Introduction}

In the hot core of fusion plasmas electron cyclotron wave losses are known to be an essential contribution to the electron power balance and, hence, affect the electron temperature profile (see, e.g., Refs. $[1,2]$ ). For plasma modelling purposes it is therefore important to be able to calculate the local net electron cyclotron (EC) wave power loss $\mathrm{dP}(\mathbf{r}) / \mathrm{dV}$ from the plasma efficiently and with good accuracy. This implies solving a non-local problem, viz., one has to integrate the equation of radiative transfer (see, e.g., Ref. [3] and in particular Section 2.2.1) to obtain the specific intensity $I^{(\sigma)}(\mathbf{r}, \omega, \hat{\mathbf{s}})$ of the ordinary $(\sigma=\mathrm{O})$ and of the extraordinary $(\sigma=\mathrm{X})$ radiation mode as a function of position $\mathbf{r}$, frequency $\omega$ and propagation direction $\hat{\mathbf{s}}$ of the ray, to be used in the expression

$$
\frac{d P(\mathbf{r})}{d V}=\sum_{\sigma=X, O} \int_{\omega_{\min }^{\sigma}}^{\infty} d \omega \int d^{2} \Omega_{\hat{\mathbf{s}}} \alpha^{(\sigma)}(\mathbf{r}, \omega, \hat{\mathbf{s}})\left[I_{b b}^{(\sigma)}(\mathbf{r}, \omega)-I^{(\sigma)}(\mathbf{r}, \omega, \hat{\mathbf{s}})\right]
$$

for the local net EC wave power loss as valid for plasmas in thermodynamic equilibrium [1]. In Eq. (1), the lower limit of the $\omega$-integration, $\omega_{\min }^{\sigma}$, accounts for the frequency cut-off of the mode $\sigma$, $d^{2} \Omega_{\hat{\mathbf{s}}}$ denotes the element of solid angle around the ray direction $\hat{\mathbf{s}}, \alpha^{(\sigma)}(\mathbf{r}, \omega, \hat{\mathbf{s}})$ is the absorption coefficient and $I_{b b}^{(\sigma)}(\mathbf{r}, \omega) \equiv\left(N_{r}^{(\sigma)}\right)^{2} \omega^{2} T(\mathbf{r}) / 8 \pi^{3} c^{2}$ is the blackbody intensity for the (local) electron temperature $T(\mathbf{r})$ with $N_{r}^{(\sigma)}$ the ray refractive index.

In order to calculate $\mathrm{dP} / \mathrm{dV}$ according to Eq.(1), an iterative numerical approach is required in which, to achieve satisfactory efficiency and accuracy, the determination of the absorption coefficient must be done in an appropriate way. While a numerical solution of the (quasi-exact) local dispersion relation [4] provides $\alpha^{(\sigma)}$ with excellent precision, this method is very computer time-consuming. Using instead an explicit analytic expression for $\alpha^{(\sigma)}$ appears therefore attractive. In Ref. [5] the accuracy of

\footnotetext{
${ }^{4}$ This article is dedicated to the memory of Ignazio Fidone

${ }^{5}$ Present address: EFDA, Max-Planck-Institut für Plasmaphysik, 85748 Garching, Germany
} 
a quasi-exact $(\mathrm{QE})$ analytical form for $\alpha^{(\sigma)}$ (obtained using Bateman's expansion for the product of two Bessel functions and containing a sum over cyclotron harmonics) and of the quite compact asymptotic expressions given earlier by Trubnikov [6] and by Robinson [7] has been assessed. As a result, the $\mathrm{QE}$ form turned out to provide an excellent approximation to $\alpha^{(\sigma)}$ but still requires considerable computation time; on the other hand, Trubnikov's and Robinson's less accurate, but simpler expressions, allow for a significantly shorter calculation time. Overall, it was found that Robinson's asymptotic form yields a reasonable approximation to the EC wave absorption coefficient (significantly better than Trubnikov's) in most of the parameter range of importance for evaluating the $\mathrm{EC}$ wave power loss in large hot fusion plasmas.

In this paper a comparison of the three above forms for the absorption coefficient in view of the accuracy achieved and of the computer time needed when calculating the local net EC wave power loss $\mathrm{dP} / \mathrm{dV}$ in ITER-like plasmas is presented, using in Eq. (1) the exact analytical solution of the equation of radiative transfer for $I^{(\sigma)}(\mathbf{r}, \omega, \hat{\mathbf{s}})$ valid for a system of circularly cylindrical symmetry with specular wall reflection properties as given in Refs. $[1,8]$. The numerical scheme adopted (the code EXACTEC) is the one devised for the analysis reported in Ref. [1]. For reference, Robinson's expression for the EC absorption coefficient is reproduced in an Appendix.

\section{Model Comparison}

We adopt ITER-like device parameters, viz., an effective plasma minor radius $a_{\text {eff }}=2.6 \mathrm{~m}$ and a toroidal magnetic field $\mathrm{B}=5.3 \mathrm{~T}$. Various fixed plasma conditions (temperatures, densities and radial profiles) have been considered. The profiles of the electron density and temperature were taken in the form $n=n_{0}\left(1-\rho^{2}\right)^{\gamma_{\mathrm{n}}}$ and $T=T_{0}\left(1-\rho^{\beta_{\mathrm{T}}}\right)^{\gamma_{\mathrm{T}}}$, respectively, with $\rho=\mathrm{r} / a_{\mathrm{eff}}$ the normalised radial coordinate; for the peak density $n_{0}$ typical values as anticipated for ITER operation, viz., $0.7 \times 10^{20}$ or $1.1 \times 10^{20} \mathrm{~m}^{-3}$ were adopted, while the peak temperature $T_{0}$ was varied between 5 and $45 \mathrm{keV}$. The density profile was assumed to be flat over most of the plasma radius $\left(\gamma_{n}=0.1\right)$. For the temperature profile mainly two cases were considered: a profile flat in the core plasma with a quite steep gradient around $\rho=0.6$ which simulates conditions as anticipated for steady-state operation using noninductive current drive ( $\gamma_{\mathrm{T}}=8.0, \beta_{\mathrm{T}}=5.4$; in the following referred to as "advanced" profile) and a "parabola-like" profile (characterized by $\gamma_{\mathrm{T}}=1.5, \beta_{\mathrm{T}}=2.0$ ). For further analysis, flat profiles of both temperature and density $\left(\gamma_{n}=\gamma_{\mathrm{T}}=\beta_{T}=0\right)$ were also addressed. The wall reflection coefficient $R_{w}$ was varied between 0 and 0.98 with most of the assessment done for $R_{w}=0.8$.

Figures 1 to 4 illustrate the results obtained presenting four typical cases, viz., peak electron temperatures of 45 and $22.5 \mathrm{keV}$ both for the "advanced" and the "parabola-like" profile fixing $n_{0}=0.7 \times 10^{20} \mathrm{~m}^{-3}$ and $R_{w}=0.8$. Part (a) of each figure shows the radial dependence of the net EC wave power loss $\mathrm{dP} / \mathrm{dV}$ as calculated using the quasi-exact $(\mathrm{QE})$, Robinson $(\mathrm{R})$ and Trubnikov $(\mathrm{T})$ expressions for the absorption coefficient as well as, for the most significant hot inner plasma (typically for $\rho \leq 0.6<$ Note to the Editor: the correct sign should be "smaller than about " $>$ ), the relative deviation

$$
\Delta \equiv \frac{(d P / d V)_{A}-(d P / d V)_{Q E}}{(d P / d V)_{Q E}}
$$

of $\mathrm{dP} / \mathrm{dV}$ as obtained applying the Robinson $(\mathrm{A}=\mathrm{R})$ and the Trubnikov $(\mathrm{A}=\mathrm{T})$ asymptotic expressions from the quasi-exact value. In Part (b) the comparison (in relative terms) of the computer times required to calculate $\mathrm{dP} / \mathrm{dV}$ at a given radial position is presented for each of the three models, together with the average $\langle\tau\rangle$ over all iteration steps, necessary for the computation of the triple integral of Eq. (1) with a given accuracy at that position, of the times $\tau$ required to do the intervening recalculations of the values of the absorption coefficients $\alpha^{(\sigma)}(<\tau>$ being obtained by dividing the total calculation time by the total number of iterations carried out; note that, effectively, iterations are necessary not only for computing the triple integral as such but also to evaluate the specific intensities 
$I^{(\sigma)}(\mathbf{r}, \omega, \hat{\mathbf{s}})$ as well as to calculate the optical distances intervening in this latter evaluation) for the three models.

\section{Discussion and Conclusions}

As a result, from Figures 1(a) to 4(a) it is seen that also for the evaluation of the profile of the net EC wave power loss $\mathrm{dP} / \mathrm{dV}$ Robinson's asymptotic form, in fact, yields a significantly more accurate result than Trubnikov's expression. Effectively, in the hot inner plasma (where $\mathrm{dP} / \mathrm{dV}$ is large) using Robinson's form leads to an overestimate of $\mathrm{dP} / \mathrm{dV}$ by less than $5 \%$ whereas Trubnikov's approximation underestimates $\mathrm{dP} / \mathrm{dV}$ by about $15 \%$. This applies as long as the local electron temperature is larger than about $15 \mathrm{keV}$ (that is, for all temperatures for which $\mathrm{EC}$ wave power losses may matter in the electron power balance). Therefore, if the plasma is hotter, the above quantification is valid for a wider radial range (e.g., for $\rho \leq 0.6<$ Note to the Editor: the correct sign should be "smaller than about" $>$ for a peak temperature $T_{0}$ of $45 \mathrm{keV}$, and for $\rho \leq 0.4<$ Note to the Editor: the correct sign should be "smaller than about" $>$ for $T_{0}=22.5 \mathrm{keV}$ ), independently of details of the temperature profile. In cooler plasmas, and specifically in the cooler outer layers, the relative deviation, from the $\mathrm{QE}$ result, of $\mathrm{dP} / \mathrm{dV}$ obtained with Robinson's form tends to increase and to become larger than that caused by applying Trubnikov's approximation. It must be noted, however, that in absolute terms these deviations remain small and the profile of $\mathrm{dP} / \mathrm{dV}$ is quite well reproduced using the approximate expressions also in the outer plasma. The impact of changing the plasma density and/or the wall reflection coefficient is negligibly small.

As far as the total computer time necessary for the calculation of $\mathrm{dP} / \mathrm{dV}$ at a given radial position is concerned, Figures 1(b) to 4(b) show that this is typically reduced by two orders of magnitude when using the approximate forms rather than the QE formula for the absorption coefficients. There is no significant difference between Robinson's and Trubnikov's approximation in this respect although there is a slight tendency of Trubnikov's model to be faster in the hot plasma core (typically up to a factor of about 1.5). The radial structure of the total computation time, to a large extent (see below), is due to the number of iterations necessary to reach the required accuracy in calculating the integral of Eq. (1) for $\mathrm{dP} / \mathrm{dV}$ at a given position. A more direct measure for the computer time requirements of the different models for the absorption coefficients is provided by the average computer time $<\tau>$ spent per iteration step. A striking result is that $<\tau>$ for both Robinson's and Trubnikov's approximation is independent of all local and global plasma parameters with Robinson's form necessitating on average 25 to $30 \%$ more time per iteration step than Trubnikov's. If the QE expression for the absorption coefficients is employed, on the other hand, there is a clear dependence of $\langle\tau\rangle$ on electron temperature $T$ : the higher is $T$ the longer is the average computer time per iteration step and that time exceeds the one using Robinson's form by about a factor of 5 for low temperatures (in the $5 \mathrm{keV}$ range) and by about a factor of 30 for temperatures approaching $45 \mathrm{keV}$. Also, there is a non-local effect of the temperature profile: the wider is the hot core of the plasma the larger is $\langle\tau\rangle$ overall as seen, e.g., from comparing the results for the "advanced" profile with those of the parabola-like one. This tendency reflects the fact that for higher temperature the EC wave spectrum is broader and, hence, in the QE expression a larger number of harmonic contributions have to be summed up. Inversely, the plasma density and the wall reflection coefficient do not have a sizeable effect either in this case.

In comparing the results for $\langle\tau\rangle$ with those for the total computation time it is apparent that both the radial position and the model employed for the absorption coefficients influence the total number of steps necessary in the iteration schemes used to calculate $\mathrm{dP} / \mathrm{dV}$ according to Eq. (1): with increasing $\rho$ the growing geometrical complexity of the ray paths (that tends to increase the number of iterations) and, for realistic profiles, the reduced interaction of the wave field with the electrons for lower temperature (which facilitates the computation) do compete with specific problems related to the profile of $\mathrm{dP} / \mathrm{dV}$ appearing at particular positions (e.g., where $\mathrm{dP} / \mathrm{dV}$ changes sign), whereas using the QE formula generally causes a considerable enhancement of the number of iteration steps required due to the more complex structure of the wave spectrum present in this model. 
In conclusion, Robinson's asymptotic form for the EC absorption coefficients is, overall, the best available for the modelling of EC wave power losses in fusion plasmas: it provides, in fact, good accuracy at significantly reduced time for computation.

\section{Appendix A: Robinson's asymptotic form for the EC absorption coefficients}

Robinson's asymptotic form for the EC absorption coefficients [7], normalised to $\omega_{p}^{2} / c \omega_{c}$, that is $\alpha^{(\sigma)} /\left(\omega_{p}^{2} / c \omega_{c}\right) \equiv \bar{\alpha}^{(\sigma)}$ with $\omega_{p}$ and $\omega_{c}$ being, respectively, the electron plasma and the EC frequency, can be written [9]

$$
\bar{\alpha}^{(\sigma)}(\bar{\omega}, \theta)=\sqrt{\pi} \frac{a(\mu) \mu^{5 / 2}}{(2 \bar{\omega})^{2}} d\left(\gamma_{0}, \theta\right)|\sin \theta|\left(\gamma_{0}^{2}-1\right)^{3 / 2} p^{(\sigma)}(\bar{\omega}, \theta) e^{-\Phi\left(\gamma_{0}, \bar{\omega}, \theta\right)} ;
$$

it is assumed here that both $\bar{\omega}=\omega / \omega_{c}$ and $\mu=m c^{2} / T$ (where $m$ is the electron rest mass) are large compared to $1 ; \theta$ is the angle of wave propagation with respect to the magnetic field;

$$
a(\mu) \equiv(\pi / 2 \mu)^{1 / 2} e^{-\mu} / K_{2}(\mu)
$$

where $K_{2}(\mu)$ is the MacDonald function;

$$
d\left(\gamma_{0}, \theta\right)=\left(1+\cot ^{2} \theta / \gamma_{0}^{2}\right)^{1 / 2}
$$

$\gamma_{0}$ is the effective Lorentz factor defined by

$$
\gamma_{0}\left(\equiv \gamma_{0}(\bar{\omega} / \mu, \theta)\right)=\left[2 \frac{\bar{\omega}}{\mu}(\chi(\theta)+1)^{-1 / 3}+1\right]^{1 / 2} \text { where } \chi(\theta) \equiv \frac{9}{2} \frac{\bar{\omega}}{\mu} \sin ^{2} \theta ;
$$

$p^{(\sigma)}$ describing the effects of wave polarisation on the absorption is given by

$$
p^{(\sigma)}(\bar{\omega}, \theta)=\frac{1}{1+\left(T^{(\sigma)}\right)^{2}}\left\{\left[d\left(\gamma_{0}, \theta\right)+\frac{\cot \theta}{\gamma_{0}} T^{(\sigma)}\right]^{2}+\frac{\gamma_{0}^{2}-1}{2 \bar{\omega}} \sin \theta d^{3}\left(\gamma_{0}, \theta\right)\left(T^{(\sigma)}\right)^{2}\right\}
$$

with

$$
T^{(X)}=-\frac{1}{T^{(O)}}=\left[b+\left(1+b^{2}\right)^{1 / 2}\right]^{-1} \text { where } b \equiv \frac{\sin ^{2} \theta}{2 \bar{\omega}|\cos \theta|}
$$

and

$$
\Phi\left(\gamma_{0}, \bar{\omega}, \theta\right) \equiv \mu\left(\gamma_{0}-1\right)+2 \bar{\omega} \gamma_{0} \sum_{k=2}^{\infty} \frac{1}{(2 k-1)\left[\gamma_{0}|\sin \theta| d\left(\gamma_{0}, \theta\right)\right]^{2 k-1}}
$$

\section{References}

[1] Albajar F, Bornatici M and Engelmann F 2002 Nucl. Fusion 42670

[2] Albajar F, Bornatici M, Cortes G, Dies J, Engelmann F, Garcia J and Izquierdo J 2005 Nucl. Fusion 45642

[3] Bornatici M, Cano R, De Barbieri O and Engelmann F 1983 Nucl. Fusion 231153

[4] Granata G and Fidone I 1991 J. Plasma Phys. 45361

[5] Albajar F, Bertelli N, Bornatici M and Engelmann F 2007 Plasma Phys. Control. Fusion 4915

[6] Trubnikov B A 1979 Reviews of Plasma Physics ed M A Leontovich, vol 7 (New York: Consultants Bureau) p 345

[7] Robinson P A 1985 Plasma Phys. Control. Fusion 271037

[8] Albajar F, Bornatici M and Engelmann F 2005 Nucl. Fusion 45 L9

[9] Bertelli N, Bornatici M and Engelmann F 2005 Phys. Letters A 347114 (Erratum 2006 Phys. Letters A 350 431) 

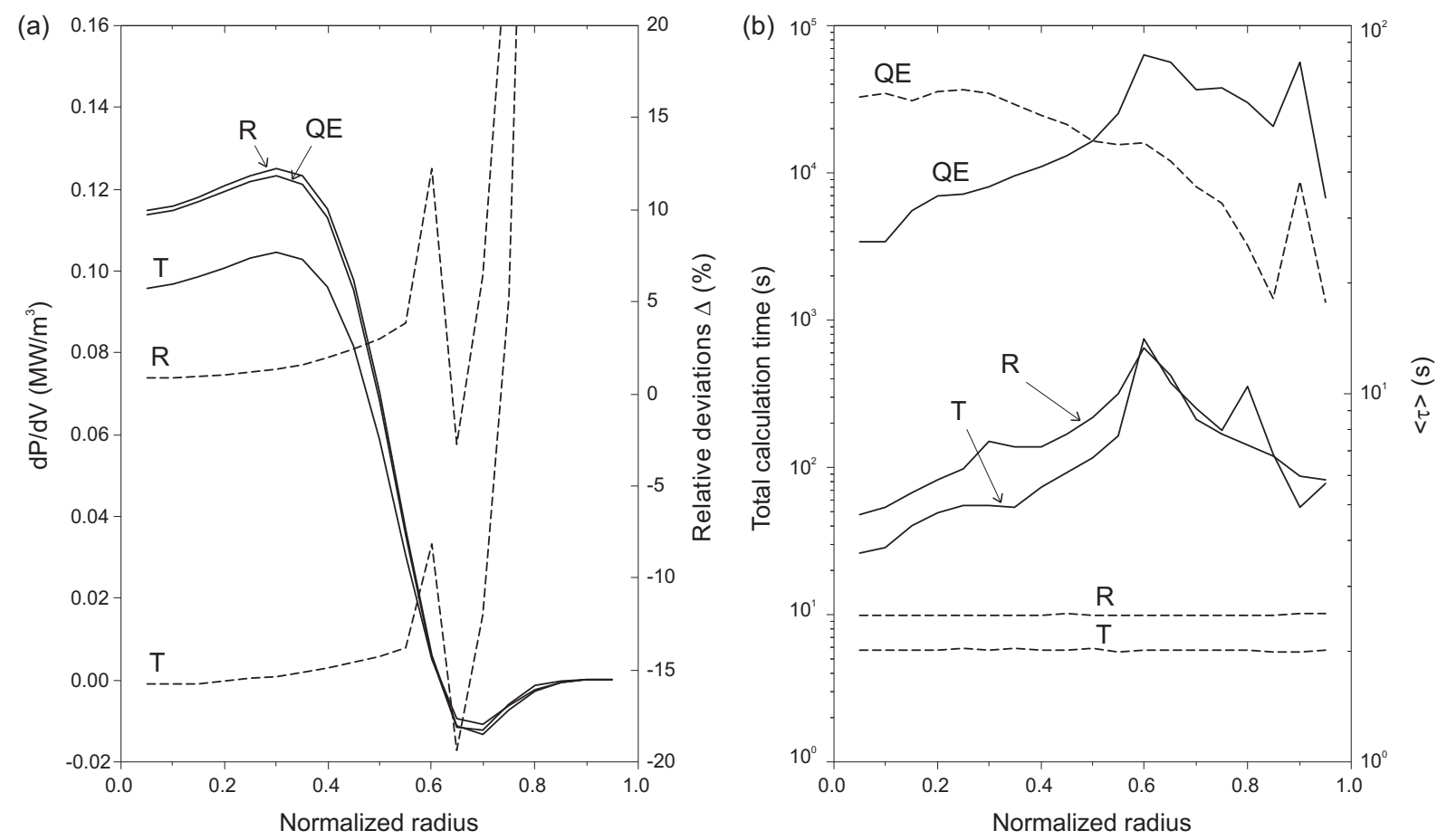

Figure 1. "Advanced" temperature profile, peak electron temperature of $45 \mathrm{keV}$, peak plasma density of $0.7 \times 10^{20} \mathrm{~m}^{-3}$ and wall reflection coefficient $R_{w}=0.8:$ (a) radial profile of the net EC wave power loss $\mathrm{dP} / \mathrm{dV}$ of the plasma electrons (full curves) for the quasi-exact (QE), Robinson (R) and Trubnikov (T) forms of the EC absorption coefficient, and relative deviations $\Delta$ of $\mathrm{dP} / \mathrm{dV}$ as evaluated with the Robinson and the Trubnikov forms from the QE result (dashed curves); (b) total calculation times necessary to evaluate one radial value of $\mathrm{dP} / \mathrm{dV}$ in arbitrary units (full curves) as a function of radial position and average $\langle\tau\rangle$ of the times employed for the computation of the values of the absorption coefficients in each of the iteration steps necessary in the evaluation of that value of $\mathrm{dP} / \mathrm{dV}$ (see text; dashed curves), for the three models. 

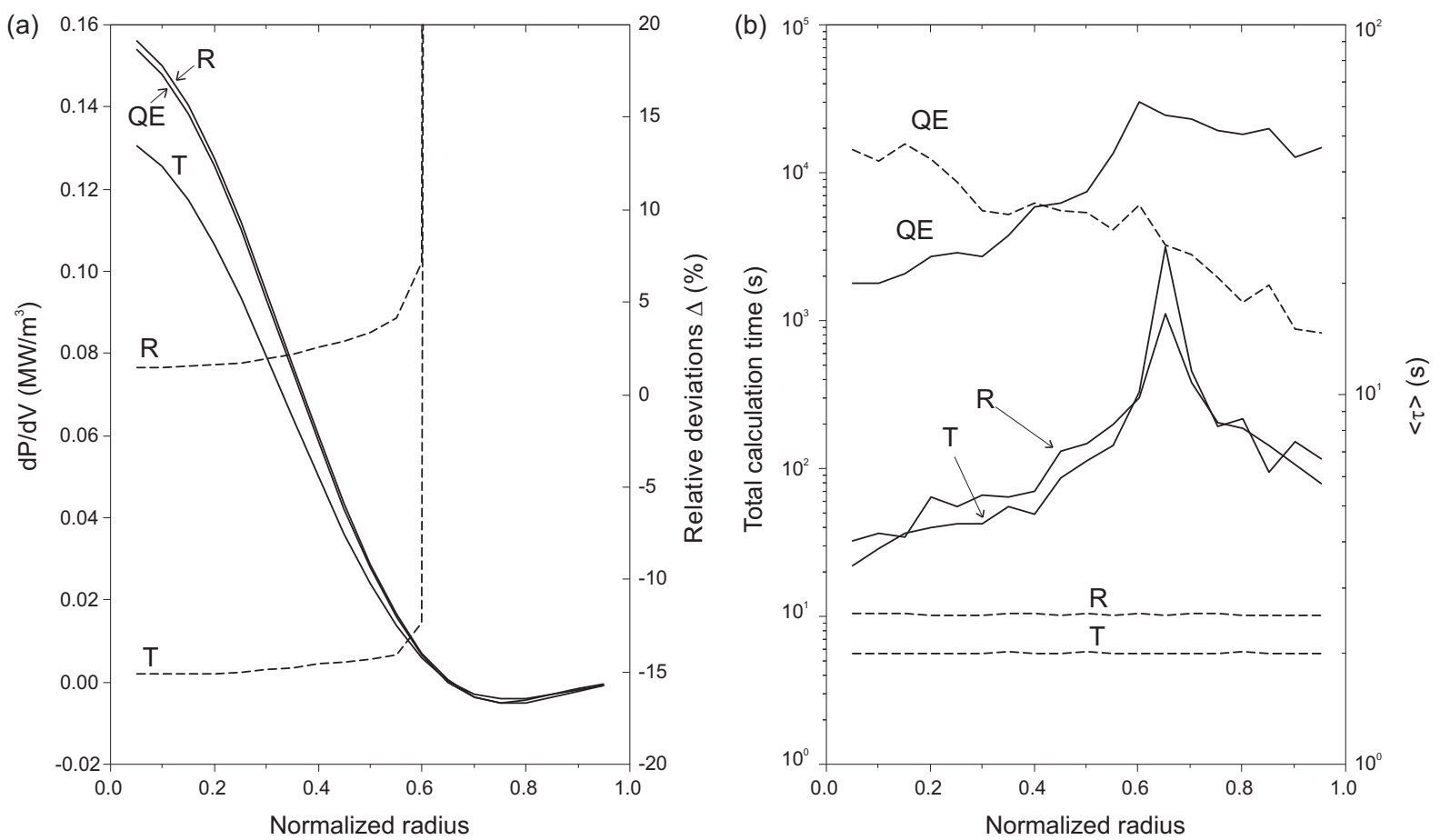

Figure 2. Same as Figure 1 for a parabola-like temperature profile. 

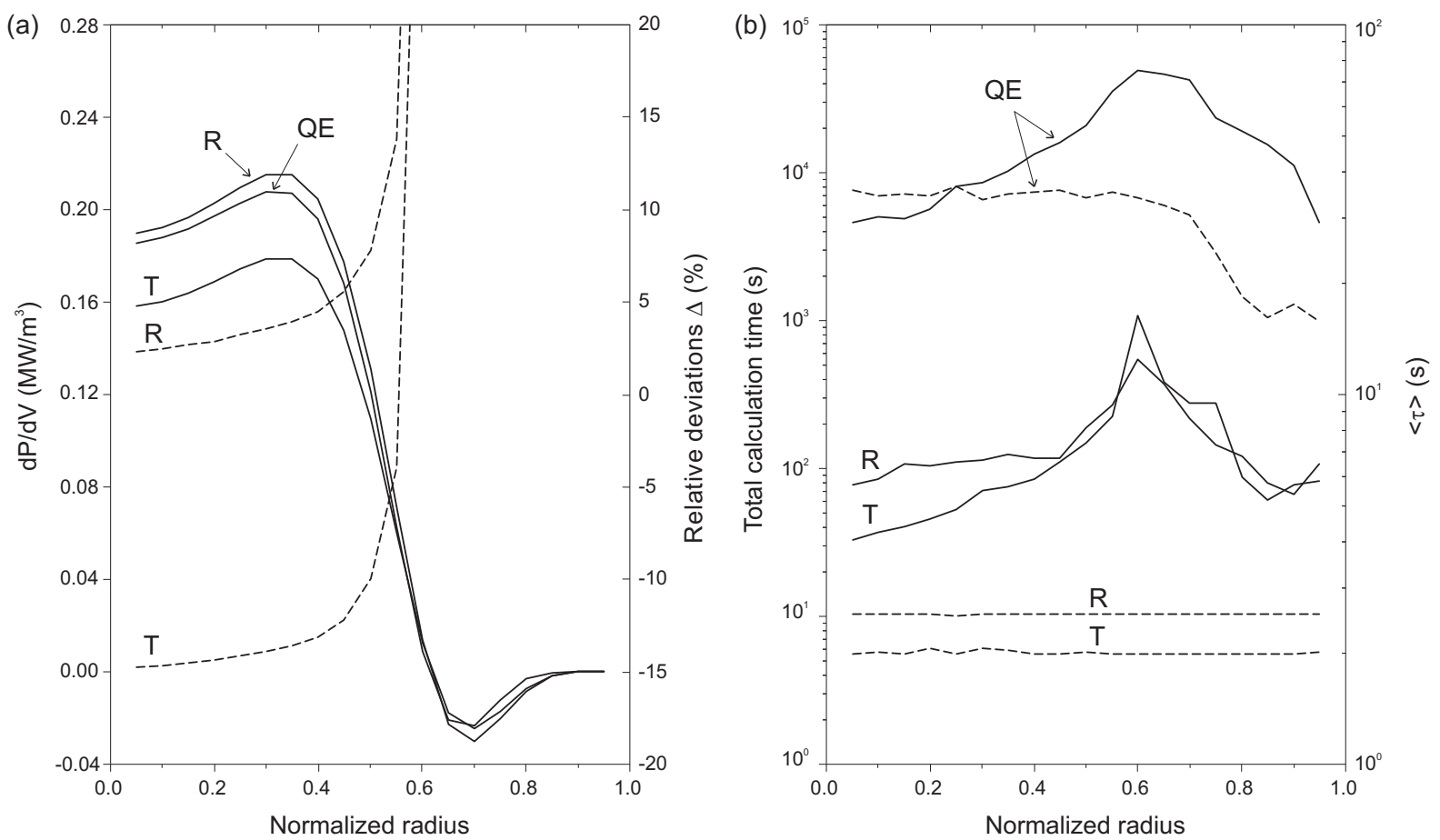

Figure 3. Same as Figure 1 for a peak electron temperature of $22.5 \mathrm{keV}$. 

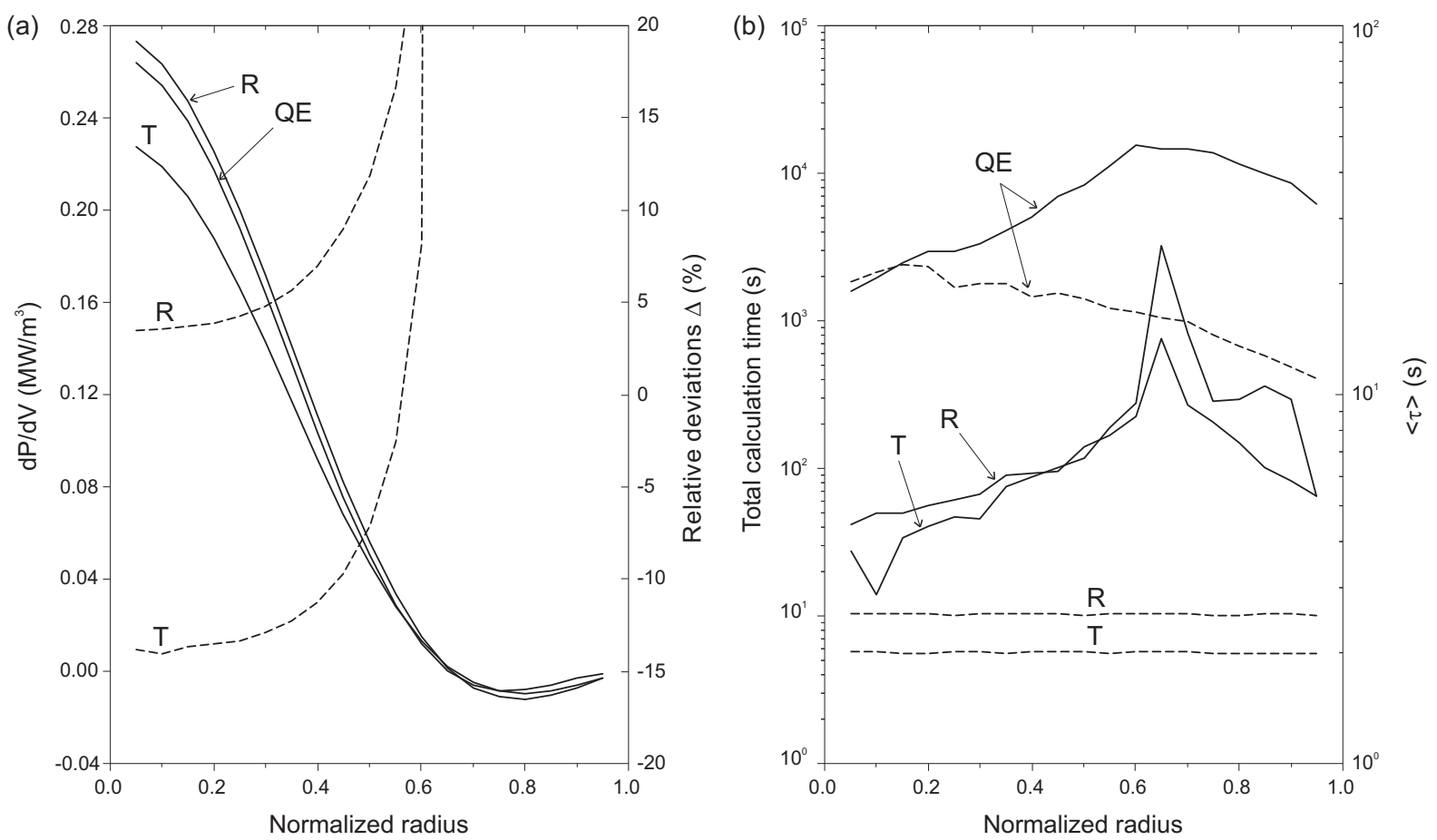

Figure 4. Same as Figure 1 for a parabola-like temperature profile with a peak temperature of $22.5 \mathrm{keV}$. 